\title{
OPINION
}

\section{The Return of the Measles to the Low Countries: A Legal-Philosophical Exploration}

\author{
Roland Pierik
}

The introduction of vaccines against infectious diseases has been one of the most important contributions to public health of the last century. Diseases like smallpox, polio, measles, mumps, whooping cough, and rubella were far and away the major killers of human beings until the beginning of the twentieth century. Nowadays, these diseases have been dramatically reduced or even eliminated, as a result of large-scale vaccination programs.

A very large majority of parents voluntarily enrol their children because they are convinced by the beneficial effects of vaccination. At the same time, since the introduction of these programs certain groups of parents have persistently refused to vaccinate their children. Until recently, non-vaccination in the Netherlands was mainly confined to 'pietistic reformed' protestant communities (bevindelijk gereformeerden) in the Bible Belt. However, in the last two decades, a broader anti-vaccination movement has emerged. This is a multifaceted movement, including anthroposophists, homeopaths and adherents of 'natural' and 'alternative healing.' They question the self-evidence with which government provides and promotes large-scale vaccination programs. Some argue that diseases such as measles could - in the case of otherwise healthy children - contribute to mental growth and to immunity building. This, so they argue, provides someone with greater resilience against diseases like cancer and allergies later in life. Others emphasise the negative effects of vaccines, because, so they argue, they contain dangerous toxic chemicals and overwhelm the immune system of young children.

The most famous example of such a backlash against vaccination programs is the MMR-vaccine causes autism-controversy a decade ago, in the wake of the publication of Andrew Wakefield's notorious 1998 paper in The Lancet. ${ }^{1}$ By now, the claim is debunked, the paper is retracted, and the author is stripped of his medical license and academic reputation. ${ }^{2}$ Still, the suggested vaccine-autism link remains 'the most damaging medical hoax of the last 100 years.' 3 The claim was widely reported in the media and went viral on anti-vaccination websites. It resulted in a sharp drop in the vaccination rates in the UK and Ireland, followed by an significantly increased incidence of measles and mumps, and ending in severe

1 The MMR-vaccine combines vaccines against measles, mumps and rubella.

2 The British General Medical Council concluded that Wakefield acted 'dishonestly and irresponsibly' while the British Medical Journal dismissed the research as an 'elaborate fraud.'

3 Dennis Flaherty, 'The Vaccine-Autism Connection: A Public Health Crisis Caused by Unethical Medical Practices and Fraudulent Science,' Ann Pharmacother 45(10) (2011): 1302-1304. 
and permanent injuries to young children and several deaths. Such incidents undermine herd immunity: the phenomenon that once a critical portion of a community is immunized against a contagious disease, the virus can no longer circulate in the population with the effect that the disease cannot gain a foothold in that society. Medical experts argue that we are about to reach a tipping point in which herd immunity can no longer be guaranteed. And indeed, we can observe a growing number of outbreaks of infectious diseases in recent years, especially the measles in Europe and whooping cough in North America. ${ }^{4}$

In the Netherlands, vaccination is neither obligatory nor required for day care or school entrance. The Dutch Government tries to nudge parents into vaccination by making vaccinations available free of charge, and through a well-organized state-immunization program (Rijksvaccinatieprogramma). The most recent occurrence of these diseases was a measles outbreak in the Bible Belt in May 2013. Almost 200 of the infected persons (all unvaccinated) were admitted to hospitals with severe symptoms like pneumonia and encephalitis. In October 2013 a seventeen years' old unvaccinated girl died from the disease. These examples make clear that measles is anything but the 'nothing disease' as some would like to present it.

Since such outbreaks were up to now mainly confined to pietistic communities on the Bible Belt, they did not attract much interest of legal scholars and legal philosophers. Such incidents were considered as an ineradicable 'folklore' within protestant communities that did not affect community at large.

However, in March 2014 a case of cross-infection to a child outside the non-vaccination community generated quite some turmoil in Dutch media. In a day care centre, a consciously unvaccinated older child infected three babies with the measles. ${ }^{5}$ One six months' old baby fell seriously ill: he spent a few days in intensive care and nearly died. Although he was enrolled in the vaccination program, he was not yet protected because the first vaccination against the measles is only given in the fourteenth month. In an interview with a major Dutch newspaper the mother stated: 'They took a gamble with the health of my child. I cannot stop them from deciding not to vaccinate their children. But don't send your unvaccinated children to a nursery in which young babies are crawling around who, because of their young age have not yet been vaccinated.' 6

Such cross-infections will mainly occur in day care centres, because unprotected babies under 14 months share facilities with children up to 12 years old. The latter are usually much more adventurous and mobile than babies and therefore have much more chance to contract the disease. Cross-infection in day care centres differs normatively from infection within a family, where parents are respon-

4 Steve P. Calandrillo, 'Vanishing Vaccinations: Why Are So Many Americans Opting out of Vaccinating Their Children?,' University of Michigan Journal of Law Reform 37(2) (2004): 353-440, at 361, 429. For a staggering overview of recent outbreaks of vaccine-preventable diseases see: http://www.cfr.org/interactives/GH_Vaccine_Map/\#map.

5 In the Netherlands it is quite common that babies from three months onwards attend day care centres.

6 de Volkskrant, 15 March 2014. 
sible for the protection of all children; or infection in schools, where all children are above the age of four and can only be infected when parents chose to forgo vaccination.

This cross-infection generated an unprecedented situation for day care centres because it generated a diametrical opposition between two categories of parents. Parents who endorse vaccination want to be sure that their young children are not exposed to the risk of infection with the measles before they can receive the vaccine. Parents who object to vaccination will claim that that their choice is constitutionally protected by the freedom of religion and should not affect admittance to day care.

The Dutch umbrella-organization for day care centres (Brancheorganisatie Kinderopvang) states that they cannot refuse children on the basis of choices made by their parents. They argue that only the state legislature can decide on the question whether sectors like day care centres, schools or sports clubs can refuse unvaccinated children, since the refusal would affect the constitutional rights of parents. ${ }^{7}$ In a response to parliamentary questions, Minister Asscher (Social Affairs and Employment) arrives at a similar conclusion: 'There is no legal basis for an overall refusal of unvaccinated children to childcare facilities; such a general refusal could result in a form of indirect discrimination against parents on grounds of religion or philosophy of life.'

The Minister is right that such an overall refusal of unvaccinated children is unwarranted, because it seems to be disproportionally restrictive towards parents who prefer not to vaccinate their children. At the same time, vaccinating parents can, in the current unregulated situation, make a legitimate claim that their concerns are not taken seriously. Their not yet vaccinated babies run the risk of infection via older unvaccinated children. And given the prevalent drop in the vaccination rates, it is not unlikely that such cross-infections will occur more often in the near future, and that they might eventually lead to a fatal accident.

Interestingly enough, Minister Asscher trivializes that risk in the aforementioned letter: 'outside the nursery children could also come into contact with unvaccinated individuals. The number of infections through nurseries is very limited in comparison to infections within the family or other social contacts such as family gatherings, travel by public transport and foreign travel.'

But this argument is beside the point. For one thing: yes, it is correct that most infections occur through family and social contacts. But these are usually interactions and infections within unvaccinated communities. Indeed, pietistic reformed communities are so vulnerable for outbreaks precisely because of their low vaccination rates and because they usually live in geographical clusters. Such closeknitted communities have much interaction through schools, churches, and communal life - think of the regional comprehensive schools, based on pietistic foundations, that serve many communities in the Bible Belt. Indeed these are first and foremost infections within the non-vaccinated community, whereas the

7 Formal statement of 17 March 2014: http://www.kinderopvang.nl.

8 Letter of 11 April 2014, no. 2014-0000050688. 
current discussion concerns cross-infections to children outside the non-vaccinated community.

Moreover, day care centres have a special responsibility. They are formal institutions with the sole aim to temporarily take over the responsibility for children from parents. This makes them more accountable for potential risks than a family member would be during a casual sleepover. This is why nurseries have comprehensive safety protocols and why, for example, their cradles seem to be ten times as sturdy and secure as those at home. Moreover, the contract between parents and the day care centre and the safety protocols might make the latter legally liable if an infection in the day care centre would eventually lead to a fatal accident.

How can the interests of vaccinating and non-vaccinating parents be aligned? As an alternative to the actual unregulated situation and the proposed general prohibition of unvaccinated children I propose a third option. Individual branches can choose to either require or not that children be vaccinated. Some day care centres will make vaccination mandatory while others do not. In this context, vaccination becomes one of the considerations that parents must balance against others in the choice for a specific centre: travel distance, opening hours, price, etc. Such an approach might provide a suitable balance between the interests of the two categories of parents involved in this discussion. Those parents who want their babies protected against contamination with diseases like the measles will opt for a centre that requires that all children admitted participate in the stateimmunization program. Parents who object to vaccination can choose a day care centre in which (full) participation in the program is not required. However, this choice would disable them to file a tort claim in the event that their child gains such an infectious disease. They had the possibility to choose for a safer day-care environment but they consciously dismissed that option. The market can solve this issue by aligning the supply and demand of vaccination-requiring and vaccination-free day care centres. This demand for the two will not fluctuate too much over time; we can expect more centres that are open to unvaccinated children around the Bible Belt and less of them in other areas. This solution does not curtail the freedom of religion because there is no overall ban of unvaccinated children in day care centres - it could only be that they are at greater travel distance in areas in which there is not much demand for them.

This policy option might have one unfortunate effect: the level of vaccination in specific centres that accept unvaccinated children might drop to such an extent that herd immunity cannot be maintained. This might lead to a serious health risk for the (unvaccinated) children. However, to the extent that this is considered to be a serious problem, it should be solved within the non-vaccinating community. It seems to be quite unfair to raise the average rate of vaccination within day care centres by reversing the separation between vaccinated and non-vaccinated day care centres. After all, this would imply that the risk of children in non-vaccinating families is reduced by a policy-choice that would increase the risk for young children in vaccinating families.

If government wants to protect children born in non-vaccinating families, those children that are as yet unable to make a well-considered choice to refuse vaccina- 
tion, it should find ways to raise vaccination rates within the non-vaccinating community. One obvious method, as I have argued elsewhere, is to (re)consider mandatory vaccination schemes for all children. ${ }^{9}$ Such a choice would, interestingly enough, make this discussion of vaccination in day care centres redundant immediately. 